\title{
Instability of the Randall-Sundrum Model and Exact Bulk Solutions
}

\author{
Hongya Liu 巴, f and Guowen Peng ${ }^{1}$
}

\begin{abstract}
Five dimensional geodesic equation is used to study the gravitational force acted on a test particle in the bulk of the Randall-Sundrum two-brane model. This force could be interpreted as the gravitational attraction from matters on the two branes and may cause the model to be unstable. By analogy with star models in astrophysics, a fluid RS model is proposed in which the bulk is filled with a fluid and this fluid has an anisotropic pressure to balance the gravity from the two branes. Thus a class of exact bulk solutions is obtained which shows that any 4D Einstein solution with a perfect fluid source can be embedded in $y=$ constant hypersurfaces in the bulk to form an equilibrium state of the brane model. By requiring a 4D effective curvature to have a minimum, the compactification size of the extra dimension is discussed.
\end{abstract}

KEY WORDS: Higher dimensions, Brane models.

\footnotetext{
${ }^{1}$ Department of Physics, Dalian University of Technology, Dalian, 116024, People's Republic of China.

${ }^{2}$ Corresponding author. Email: hyliu@dlut.edu.cn
} 


\section{INTRODUCTION}

There is a strong interest in the possibility that our universe is a 3-brane embedded in a higher dimensional space. It has been proposed that the large hierarchy between the weak scale and the fundamental scale of gravity can be eliminated if the extra compact dimensions are large [1]. An alternative solution to the hierarchy problem, proposed by Randall and Sundrum (RS), assumes that our universe is a negative tension brane separated from a positive tension brane by a five-dimensional anti-de Sitter $\left(A d S_{5}\right)$ bulk space 2 . This does not require a large extra dimension: the hierarchy problem is solved by the special properties of the AdS space. A similar scenario to the RS one is that of Horava and Witten[3], which arises within the context of M-theory.

The RS two-brane solution satisfies the 5D Einstein equations

$$
R_{A B}-\frac{1}{2} g_{A B} R=-\frac{1}{4 M^{3}}\left\{\Lambda g_{A B}+\left[\lambda_{v i s} \delta\left(y-y_{c}\right)+\lambda_{h i d} \delta(y)\right] g_{\mu \nu} \delta_{A}^{\mu} \delta_{B}^{\nu}\right\}
$$

with a non-factorizable 5D metric being

$$
d s^{2}=W^{2}(y) \widetilde{\eta}_{\alpha \beta} d x^{\alpha} d x^{\beta}+d y^{2}
$$

Here and in the following we use signature $(-++++)$, and we use upper case Latin letters to denote $5 \mathrm{D}$ indices $(0,1,2,3,5)$ and lower case Greek letters to denote $4 \mathrm{D}$ indices $(0,1,2,3)$. In (11) and (2), the "warp" factor $W(y)$ is

$$
W(y)=e^{-k|y|}
$$

and $\lambda_{\text {vis }}, \lambda_{\text {hid }}$ and $\Lambda$ are

$$
\lambda_{\text {hid }}=-\lambda_{\text {vis }}=24 M^{3} k, \Lambda=-24 M^{3} k^{2} .
$$

In this solution, the fifth dimension has the $Z_{2}$ reflection symmetry $(x, y) \rightarrow(x,-y)$ with

$-y_{c} \leq y \leq y_{c}$. The hidden brane and the visible brane are located at $y=0$ and $y=y_{c}$ respectively. 
The instability of the RS model has received extensive studies [4,5]. In this paper, we wish to approach this subject from a different perspective. The paper is arranged as follows. In section 2 we use the 5D geodesic equations to study the instability of the model. In section 3 we introduce a $5 \mathrm{D}$ anisotropic fluid in the bulk and derive a hydrostatic equilibrium equation of the bulk fluid along the $y$-direction. In section 4 we look for exact solutions of the 5D Einstein equations. In section 5 we discuss the embedding of several well known 4D exact solutions. In section 6 we study the compactification size of the fifth dimension.

\section{GRAVITATIONAL FORCE IN THE BULK}

In this section we study the gravitational interaction between matters on the two branes of the RS model. It is known from the brane-world scenario that Standard Model (SM) particles are confined to branes while gravitons can freely propagate in the bulk. Now let us consider a test particle in the bulk. It is reasonable to expect that the motion of a bulk test particle, which is acted on by the gravitational force only, is described by the following $5 \mathrm{D}$ geodesics[6]:

$$
\frac{d^{2} x^{A}}{d \tau^{2}}+\Gamma_{B C}^{A} \frac{d x^{B}}{d \tau} \frac{d x^{C}}{d \tau}=0
$$

where $\Gamma_{B C}^{A}$ is the Christoffel symbol for the 5D metric $g_{A B}$ and $d \tau^{2}=-d s^{2}=-g_{A B} d x^{A} d x^{A}$. It is known that 5D geodesic equations (5) may yield extra 4D forces [6] [7]. In this paper, we are not going to study this kind of extra forces; we only wish to study particle's motion along the fifth direction. From (5), the 5D gravitational force can be defined as

$$
F^{A}=-\Gamma_{B C}^{A} \frac{d x^{B}}{d \tau} \frac{d x^{C}}{d \tau} .
$$

Using (2) and (3) we find that the fifth component of $F^{A}$ is

$$
\frac{d^{2} y}{d \tau^{2}}=F^{5}=-\Gamma_{B C}^{5} \frac{d x^{B}}{d \tau} \frac{d x^{C}}{d \tau}=\varepsilon k\left[1+\left(\frac{d y}{d \tau}\right)^{2}\right],
$$


where

$$
\varepsilon=\left\{\begin{array}{l}
1 \quad \text { for } \quad y>0 \\
-1 \quad \text { for } \quad y<0
\end{array}\right.
$$

So we find

$$
\begin{aligned}
& F^{5}>0 \text { for } y>0, \\
& F^{5}<0 \text { for } y<0 .
\end{aligned}
$$

This result shows that the force $F^{5}$ acting on the bulk test particle points from the hidden brane at $y=0$ to the visible brane in both $y>0$ and $y<0$ sides. So a bulk test particle will eventually move to the visible brane at $y=y_{c}$. This may cause the RS two brane model to be unstable. Firstly, we note that $y=0$ is an unstable equilibrium position while $y=y_{c}$ is a stable one. Secondly, it was argued that in sufficiently hard collisions the SM particles can acquire momentum in the extra dimensions and escape from the branes [1]. As soon as a SM particle was kicked off the hidden brane at $y=0$ into the bulk, it will be pulled by $F^{5}$ down to the visible brane at $y=y_{c}$. In this way, the distribution of matter on the two branes can not remain balanced. So we say that the hidden brane is unstable.

In the single brane $\mathrm{RS}$ model $[8]$, the $y=y_{c}$ brane approaches the AdS horizon. We find that above discussion and conclusion also valid.

It has been noted 9] that if the Minkowski metric $\widetilde{\eta}_{\alpha \beta}$ in the RS solution (2) is replaced by any Ricci flat metric $\widetilde{g}_{\alpha \beta}$ then the Einstein equations (11) are still satisfied [10. This enable people to study any 4D Einstein's vacuum solutions such as the Schwarzschild one in the RS scenario. A very interesting work of this kind is discussed in Ref. [9] where the 5D Schwarzschild solution is called the brane-world black hole, black string, or black cigar. Here we find that even in the Ricci-flat case the three equations (7)-(9) still hold. Therefore, the conclusion is the same that the $y=0$ brane for Ricci-flat metrics $\widetilde{g}_{\alpha \beta}$ is also unstable. 


\section{EQUILIBRIUM EQUATION OF THE BULK FLUID}

To resolve the instability problem, we follow others [1] [12] to introduce a 5D fluid in the bulk to balance the attraction between the two branes and to form a hydrodynamical model. This introduction would generalize the Ricci-flat metric $\widetilde{g}_{\alpha \beta}$ once more to non Ricci-flat $4 \mathrm{D}$ metrics, for which we let

$$
d s^{2}=W^{2}(y) \widetilde{g}_{\alpha \beta}\left(x^{\mu}\right) d x^{\alpha} d x^{\beta}+d y^{2},
$$

where $\widetilde{g}_{\alpha \beta}$ is the induced $4 \mathrm{D}$ metric. For the bulk matter, we use anisotropic 5D fluid model and require that the bulk fluid does not flow along the $y$-direction[12], i.e., $u^{5} \equiv d y / d \tau=0$. That is, we let

$$
\begin{aligned}
T^{A B} & =\left(\begin{array}{ll}
T^{\alpha \beta} & 0 \\
0 & P
\end{array}\right), \\
T^{\alpha \beta} & =(\rho+p) u^{\alpha} u^{\beta}+p g^{\alpha \beta},
\end{aligned}
$$

where $T^{\alpha \beta}$ is of the $4 \mathrm{D}$ perfect fluid form with $u^{\alpha} \equiv d x^{\alpha} / d \tau$. Then by using the fifth equation of the $5 \mathrm{D}$ Bianchi identities $T^{A B} ;_{B}=0$ we obtain a condition

$$
P^{\prime}=\frac{W^{\prime}}{W}(3 p-\rho-4 P),
$$

where we have used the relation $g_{\alpha \beta} T^{\alpha \beta}=3 p-\rho\left(\right.$ since $\left.u^{5}=0\right)$ and a prime stands for partial derivative with respect to $y$. This condition (12) is a constraint upon the bulk fluid which is similar to that of star models in astrophysics. Accordingly, we call (12) the hydrostatic equilibrium equation of the bulk fluid along the $y$-direction.

\section{EXACT 5D BULK SOLUTIONS}

Now the 5D Einstein equations read

$$
R_{A B}-\frac{1}{2} g_{A B} R=\frac{1}{4 M^{3}}\left\{T_{A B}-\Lambda g_{A B}-\left[\lambda_{v i s} \delta\left(y-y_{c}\right)+\lambda_{h i d} \delta(y)\right] g_{\mu \nu} \delta_{A}^{\mu} \delta_{B}^{\nu}\right\}
$$


where $T_{A B}$ takes the form (11). To solve equations (13), we firstly use the metric (10) to reduce $R_{A B}-\frac{1}{2} g_{A B} R$ to

$$
\begin{aligned}
R_{\alpha \beta}-\frac{1}{2} g_{\alpha \beta} R & =\widetilde{R}_{\alpha \beta}-\frac{1}{2} \widetilde{g}_{\alpha \beta} \widetilde{R}+3\left(W W^{\prime \prime}+W^{\prime 2}\right) \widetilde{g}_{\alpha \beta} \\
R_{\alpha 5} & =0 \\
R_{55}-\frac{1}{2} g_{55} R & =-\frac{1}{2} W^{-2} \widetilde{R}+6 W^{-2} W^{\prime 2}
\end{aligned}
$$

where $\widetilde{R}_{\alpha \beta}$ and $\widetilde{R}$ are made from $\widetilde{g}_{\alpha \beta}$. Then, by substituting (14) into (13), we obtain

$$
\begin{gathered}
\widetilde{R}_{\alpha \beta}-\frac{1}{2} \widetilde{g}_{\alpha \beta} \widetilde{R}+3\left(W W^{\prime \prime}+W^{\prime 2}\right) \widetilde{g}_{\alpha \beta}=\frac{1}{4 M^{3}}\left\{T_{\alpha \beta}-\left[\Lambda+\lambda_{v i s} \delta\left(y-y_{c}\right)+\lambda_{h i d} \delta(y)\right] g_{\alpha \beta}\right\} \\
-\frac{1}{2} \widetilde{R}+6 W^{\prime 2}=\frac{1}{4 M^{3}}(P-\Lambda) W^{2}
\end{gathered}
$$

Now we wish to know what kind of exact solutions of Eqs. (15) and (16) could fit into the RS two brane model without changing the boundary conditions (3) and (舟). So we suppose that the "warp" factor $W(y)$ and the cosmological constants $\Lambda, \lambda_{v i s}$, and $\lambda_{\text {hid }}$ take the same forms as they do in the RS solution (3) and (田). Then Eqs. (15) and (16) reduce to

$$
\begin{aligned}
\widetilde{R}_{\alpha \beta}-\frac{1}{2} \widetilde{g}_{\alpha \beta} \widetilde{R} & =\frac{1}{4 M^{3}}\left[(\rho+p) u_{\alpha} u_{\beta}+p g_{\alpha \beta}\right], \\
\widetilde{R} & =-\frac{1}{2 M^{3}} P W^{2},
\end{aligned}
$$

where we have used (11). We see that the left-hand sides of these two equations are functions of the $4 \mathrm{D}$ coordinates $x^{\mu}$ only. So we wish somehow to arrange to have the right-hand sides of the two equations also depend on $x^{\mu}$ only. To do this, let us define the induced $4 \mathrm{D}$ velocity by $\widetilde{u}^{\alpha} \equiv d x^{\alpha} / d \widetilde{\tau}$, where $d \widetilde{\tau}^{2}=-\widetilde{g}_{\alpha \beta} d x^{\alpha} d x^{\beta}$. So $u^{\alpha} \equiv d x^{\alpha} / d \tau=(d \widetilde{\tau} / d \tau) \widetilde{u}^{\alpha}$. Since $u^{5}=0$ we have

$$
u^{\alpha}=W^{-1} \widetilde{u}^{\alpha}, \quad u_{\alpha}=W \widetilde{u}_{\alpha}
$$


Using this into (17) gives

$$
\widetilde{R}_{\alpha \beta}-\frac{1}{2} \widetilde{g}_{\alpha \beta} \widetilde{R}=\frac{1}{4 M^{3}} W^{2}\left[(\rho+p) \widetilde{u}_{\alpha} \widetilde{u}_{\beta}+p \widetilde{g}_{\alpha \beta}\right]
$$

Note that $\widetilde{u}_{\alpha}$ and $\widetilde{g}_{\alpha \beta}$ depend on $x^{\mu}$ only. So from this equation and equation (18) we obtain

$$
\rho=b W^{-2} \widetilde{\rho}, \quad p=b W^{-2} \widetilde{p}, \quad P=b W^{-2} \widetilde{P}
$$

where $b$ is a constant, $\rho, p$ and $P$ satisfy the following condition

$$
2 P=3 p-\rho
$$

and $\widetilde{\rho}, \widetilde{p}$ and $\widetilde{P}$ are functions of $x^{\mu}$ only. Thus, with

$$
b=32 M^{3} \pi G_{4}
$$

we have successfully brought equations (17) to the form of the standard 4D Einstein's equations with a perfect fluid source:

$$
\begin{aligned}
\widetilde{R}_{\alpha \beta}-\frac{1}{2} \widetilde{g}_{\alpha \beta} \widetilde{R} & =8 \pi G_{4} \widetilde{T}_{\alpha \beta}, \\
\widetilde{T}_{\alpha \beta} & =(\widetilde{\rho}+\widetilde{p}) \widetilde{u}_{\alpha} \widetilde{u}_{\beta}+\widetilde{p} \widetilde{g}_{\alpha \beta} .
\end{aligned}
$$

Note that equation (22) plays the same role as the hydrostatic equilibrium equation (12), and (12) is satisfied automatically. It is also noticed that some results, such as relations (21) and (22), are recoveries of previous works 12, and is compatible with global constraints known as the brane world sum rules 13.

Here, we can call $\widetilde{T}_{\alpha \beta}$ in (24) the effective $4 \mathrm{D}$ energy-momentum tensor. Many discussions concerning this kind of effective or induced energy momentum tensor can be found in the induced matter theory [14] in which the 4D matter could be a consequence of the dependence of the 5D metric on the extra dimension. This is also true in brane models in which if the $5 \mathrm{D}$ metric is independent of the extra dimension, then the brane is void of matter. Detailed 
discussions for the relationship between the induced-matter and the brane-world theories can be found in Ref. [15.

We also wish to emphasis that our derivation for solutions (21) and (22) is very general. The only restriction is that the $5 \mathrm{D}$ bulk energy-momentum tensor $T^{A B}$ should take a fluid form (11). Since (24) are just the 4D Einstein equations, we can conclude that any known $4 \mathrm{D}$ exact solution, which has a perfect fluid as source, can be embedded in 4D hypersurfaces of the bulk to generate a $5 \mathrm{D}$ exact solution of the $5 \mathrm{D}$ equations (13), with $5 \mathrm{D}$ metric as in (10), the "warp" factor $W(y)$ and the cosmological constants $\Lambda, \lambda_{\text {vis }}, \lambda_{\text {hid }}$ as in (3i) and (44), and $\widetilde{\rho}, \widetilde{p}, \widetilde{P}$ satisfy relations (21) and (22).

\section{EMBEDDING OF 4D EINSTEIN SOLUTIONS}

It is well known that most 4D exact solutions of general relativity have used a perfect fluid as source, such as the standard FRW cosmological solutions and various exterior and interior solutions for various rotating and non-rotating neutral stars. Using the relations obtained in section 4, all these solutions can easily be embedded in the RS model to form 5D exact solutions without changing the RS boundaries. For example, the 5D FRW cosmological solutions are

$$
d s^{2}=e^{-2 k|y|}\left[-d t^{2}+a^{2}(t)\left(\frac{d r^{2}}{1-k^{\prime} r^{2}}+r^{2} d \Omega^{2}\right)\right]+d y^{2},
$$

where $k^{\prime}$ is the $3 \mathrm{D}$ curvature index $\left(k^{\prime}= \pm 1,0\right), d \Omega^{2} \equiv d \theta^{2}+\sin ^{2} \theta d \varphi^{2}$, and

$$
\begin{aligned}
\left(\frac{d a}{d t}\right)^{2}+k^{\prime} & =\frac{8 \pi G_{4}}{3} \widetilde{\rho} a^{2}, \\
a^{3} \frac{d \widetilde{p}}{d t} & =\frac{d}{d t}\left[(\widetilde{\rho}+\widetilde{p}) a^{3}\right], \\
\rho & =\left(G_{4} / G_{5}\right) e^{2 k|y|} \widetilde{\rho}(t), \\
p & =\left(G_{4} / G_{5}\right) e^{2 k|y|} \widetilde{p}(t), \\
2 P & =\left(G_{4} / G_{5}\right) e^{2 k|y|}[3 \widetilde{p}(t)-\widetilde{\rho}(t)],
\end{aligned}
$$


where $8 \pi G_{5}=\left(4 M^{3}\right)^{-1}$. From these we see that $\rho, p$ and $P$ increase exponentially when $y$ tends from the hidden brane at $y=0$ to the visible brane at $y=y_{c}$.

As a second example, we write down the Schwarzschild-AdS $\mathrm{S}_{5}$ solution in the following:

$$
d s^{2}=e^{-2 k|y|}\left[-U(r) d t^{2}+U(r)^{-1} d r^{2}+r^{2} d \Omega^{2}\right]+d y^{2}
$$

where

$$
U(r)=1-\frac{2 G_{4} M}{r}+\frac{1}{3} \widetilde{\lambda} r^{2}
$$

and

$$
\begin{aligned}
-\tilde{\rho} & =\widetilde{p}=\frac{1}{8 \pi G_{4}} \tilde{\lambda} \\
-\rho & =p=\frac{1}{8 \pi G_{5}} e^{2 k|y|} \bar{\lambda} \\
P & =\frac{1}{4 \pi G_{5}} e^{2 k|y|} \tilde{\lambda} .
\end{aligned}
$$

So in the vicinity of the visible brane, the magnitude of the $5 \mathrm{D}$ densities $\rho, p$ and $P$ are much larger than those in the vicinity of the hidden brane.

By using known 4D exact solutions, more 5D exact solutions can be obtained easily in this way. We can show that the hydrostatic equilibrium equation (12) is satisfied by all these solutions. So all these solutions are equilibrium states of the RS model.

\section{COMPACTIFICATION SIZE OF THE FIFTH DI- MENSION}

By introducing a scalar field in the bulk, Goldberger and Wise [4 proposed a dynamics to stabilize the size of the extra dimension. The mechanism was to integrate the scalar field action over the fifth dimension to yield an effective $4 \mathrm{D}$ potential. Then it was found that this potential has a minimum which yields a compactification radius without fine tuning of 
parameters. In our case, there is no scalar field available in the bulk. If one still wish to stabilize the extra dimension, one may need look for another quantity to minimum.

For simplicity, let us consider the 5D Schwarzschild-AdS 5 solution (27)-(29). For this solution the 5D scalar curvature $R$ can be calculated by using (13), (111), (29) and the relation $8 \pi G_{5}=\left(4 M^{3}\right)^{-1}$ as

$$
R=-\frac{1}{6 M^{3}}\left\{24 M^{3} \widetilde{\lambda} e^{2 k|y|}-5 \Lambda-4\left[\lambda_{v i s} \delta\left(y-y_{c}\right)+\lambda_{h i d} \delta(y)\right]\right\} .
$$

Now we consider the geometrical part of the $5 \mathrm{D}$ action

$$
S_{\text {geo }}=\int d^{4} x \int_{-y_{c}}^{y_{c}} 2 M^{3} \sqrt{-g} R d y
$$

Substituting (30) into this equation and integrating over the fifth dimension, we obtain

$$
S_{\text {geo }}=-\frac{1}{3} \int \sqrt{-\widetilde{g}} d^{4} x\left[\frac{24}{k} M^{3} \widetilde{\lambda}\left(1-e^{-2 k y_{c}}\right)-\frac{5}{2 k} \Lambda\left(1-e^{-4 k y_{c}}\right)-4 \lambda_{\text {vis }} e^{-4 k y_{c}}-4 \lambda_{\text {hid }}\right] .
$$

Denote the expression inside the square bracket of this equation as $K$, and use (4) to eliminate $\Lambda, \lambda_{v i s}$, and $\lambda_{\text {hid }}$ in $K$, we find

$$
K \equiv-6 M^{3} \int_{-y_{c}}^{y_{c}} W^{4} R d y=\frac{24}{k} M^{3} \tilde{\lambda}\left(1-e^{-2 k y_{c}}\right)-36 M^{3} k\left(1-e^{-4 k y_{c}}\right) .
$$

This $K$ can be interpreted as an effective $4 \mathrm{D}$ curvature. Interestingly we find that this $K$ has a minimum at

$$
e^{-2 k y_{c}}=\frac{\tilde{\lambda}}{3 k^{2}}
$$

at which

$$
\frac{\partial^{2} K}{\partial y_{c}^{2}}=\frac{32}{k} M^{3} \widetilde{\lambda}^{2}>0
$$

Therefore we see that the relation (34) may provide us with a possible compactification size $y_{c}$ for the fifth dimension. 
Note that the relation (34) requires $\tilde{\lambda}$ being positive. Be aware that if $\tilde{\lambda}$ is negative, then the 5D Schwarzschild- $\mathrm{AdS}_{5}$ solution (27)-(29) becomes the 5D Schwarzschild-dS $\mathrm{S}_{5}$ solution. So the effective $4 \mathrm{D}$ curvature $K$ of the $5 \mathrm{D}$ Schwarzschild-dS $\mathrm{S}_{5}$ solution does not have a minimum.

From (28) it is reasonable to expect $\widetilde{\lambda} y_{c}^{2} \ll 1$. For instance, if $\tilde{\lambda} y_{c}^{2}=10^{-31}$, then $k y_{c} \simeq 40$. We find that this value of $y_{c}$ meets the requirement from the hierarchy problem [2].

\section{CONCLUSION}

In this paper we have studied the gravitational force field in the bulk of the RS two brane model by using the 5D geodesic equations. This force may cause the hidden brane to be unstable. To balance this force we have introduced a 5D fluid in the bulk with it's 4D part being a perfect fluid. Thus a hydrostatic equilibrium equation for the bulk fluid is derived. Meanwhile, a class of exact bulk solutions is obtained. In 4D hypersurfaces these solutions turn out to be exactly the same as the 4D Einstein equations with a perfect fluid source. Therefore, one can obtain exact 5D bulk solutions by simply embedding a suitable 4D solution in the bulk. Then we have discussed the stabilization size of the extra dimension. Further investigation is needed.

\section{ACKNOWLEDGMENTS}

This work was supported by the National Natural Science Foundation of China under grant 19975007 .

\section{References}

[1] Arkani-Hamed, N., Dimopoulos, S., and Dvali, G. (1998). Phys. Lett. B 429, 263, hepph/9803315; Arkani-Hamed, N., Dimopoulos, S., and Dvali, G. (1999). Phys. Rev. D 59, 086004 , hep-ph/9807344; Antoniadis, I., Arkani-Hamed, N., Dimopoulos, S., and Dvali, G. (1998). Phys. Lett. B 436, 257, hep-ph/9804398. 
[2] Randall, L., and Sundrum, R. (1999). Phys. Rev. Lett. 83, 3370 , hep-ph/9905221.

[3] Horava, P., and Witten, E. (1996). Nucl. Phys. B 460, 506 , hep-th/9510209; Horava, P., and Witten, E. (1996). Nucl. Phys. B 475, 94, hep-th/9603142; Witten, E. (1996). Nucl. Phys. B 471, 135 , hep-th/9602070.

[4] Goldberger, W. D., and Wise, M. B. (1999). Phys. Rev. D 60, 107505, hep-ph/9907218; Goldberger W. D., and Wise, M. B. (1999). Phys. Rev. Lett. 83, 4922 , hep-ph/9907447; Goldberger W. D., and Wise, M. B. (2000). Phys. Lett. B 475, 275, hep-ph/9911457.

[5] Csaki, C., Graesser, M., Randall, L., and Terning, J. (2000). Phys. Rev. D 62, 045015 , hep-ph/9911406; DeWolfe, O., Freedman, D. Z., Gubser, S. S., and Karch, A. (2000). Phys. Rev. D 62, 046008, hep-th/9909134; Goldberger, W., and Rothstein, I. (2000). Phys. Lett. B 491, 339 , hep-th/0007065; Luty, M. A., and Sundrum, R. (2000). Phys. Rev. D 62, 035008 , hep-th/9910202.

[6] Youm, D. (2000). Phys. Rev. D 62, 084002, hep-th/0004144.

[7] Mashhoon, B., Wesson, P., and Liu, H. (1998). Gen. Rel. Grav. 30, 555; Liu, H., and Mashhoon, B. (2000). Phys. Lett. A 272, 26 , gr-qc/0005079; Ponce de Leon, J. (2001). Phys. Lett. B 523, 311, gr-qc/0110063.

[8] Randall, L., and Sundrum, R. (1999). Phys. Rev. Lett. 83, 4690, hep-th/9906064.

[9] Chamblin, A., Hawking, S. W., and Reall, H. S.(2000). Phys. Rev. D 61, 065007 , hep-th/9909205.

[10] Brecher D., and Perry, M. J. (2000). Nucl. Phys. B 566, 151 , hep-th/9908018.

[11] Binetruy, P., Deffayet, C., and Langlois, D. (2000). Nucl. Phys. B 565, 269 , hepth/9905012. 
[12] Kanti, P., Kogan, I., Olive, K. A., and Pospelov, M. (1999). Phys. Lett. B 468, 31 , hep-ph/9909481; Kanti, P., Kogan, I. I., Olive K. A., and Pospelov, M. (2000). Phys. Rev. D 61, 106004 , hep-ph/9912266; Kanti, P., Olive K. A., and Pospelov, M. (2000). Phys. Lett. B 481, 386 , hep-ph/0002229; Kanti, P., Olive K. A., and Pospelov, M. (2000). Phys. Rev. D 62, 126004 , hep-ph/0005146; Kennedy, C., and Prodanov, E. M. (2000). Phys. Lett. B 488, 11 , hep-th/0003299; Kennedy, C., and Prodanov, E. M. (2000). Phys. Lett. B 498, 272, hep-th/0010202; Enqvist, K., Keski-Vakkuri, E., and Rasanen, S. hep-th/0007254.

[13] Gibbons, G., Kallosh R., and Linde, A. (2001). JHEP 0101, 022 , hep-th/0011225.

[14] Wesson, P.S., Space-Time-Matter (world Scientific, Singapore, 1999); Overduin, J.M. and Wesson, P.S. (1997). Phys. Rep.283, 303, gr-qc/9805018; Wesson, P.S. and Ponce de Leon (1992). J. Math. Phys. 33, 3883.

[15] Ponce de Leon, J. (2001). Mod. Phys. Lett. A, 16, 2291, gr-qc/0111011. 